\title{
PARASITOIDES NATIVOS DE RHAGOLETIS POMONELLA WALSH (DIPTERA: TEPHRITIDAE) EN TEJOCOTE CRATAEGUS SPP. EN EL CENTRO DE MÉXICO
}

\author{
Erica MuÑz-Reyes, ${ }^{1}$ J. Refugio Lomelí-Flores y Julio SÁNCHEZ-EsCudero \\ Colegio de Postgraduados. Posgrado en Entomología y Acarología. Km 36.5 carretera México- \\ Texcoco, Montecillo, Edo. De México. CP. 56230., México. \\ <ericamr@colpos.mx>
}

Muñiz-Reyes, E., J. R. Lomelí-Flores \& J. Sánchez-Escudero. 2011. Parasitoides nativos de Rhagoletis pomonella Walsh (Diptera: Tephritidae) en tejocote Crataegus spp. en el centro de México. Acta Zoológica Mexicana (n. s.), 27(2): 425-440.

RESUMEN. Rhagoletis pomonella es una de la principales plagas de tejocote en la zona central de México, donde llega a ocasionar la pérdida de $100 \%$ de la cosecha si no se realizan medidas de control. Con la finalidad de conocer el complejo de enemigos naturales asociados a esta especie, se realizaron colectas de puparios de este tefrítido en Texcoco, Edo. de México de 2007 a 2009. Los puparios se mantuvieron en condiciones controladas durante la diapausa en espera de la emergencia de parasitoides. Se colectaron 2734 puarios de los cuales sólo emergieron 1460 organismos, $68.3 \%$ fueron moscas y el restante $31.7 \%$ fueron parasitoides. Se obtuvieron e identificaron seis especies de parasitoides: Coptera sp. (Diapriidae) fue la especie más abundante (25\% del total de parasitismo) y representa un nuevo reporte de este diapríido atacando pupas de R. pomonella. Utetes near canaliculatus (Braconidae) (3.4\%), ya reportado como parasitoide de esta mosca en México; Diachasmimorpha mexicana (Braconidae) (2.3\%), no reportado para esta zona. Hemipenthes blanchardiana (Bombyliidae) $(<0.5 \%)$ representa el primer reporte de especie y familia atacando a $R$. pomonella. Finalmente se encontró un individuo de una especie de Ichneumonidae y una especie de Braconidae, que no fueron identificados. El porcentaje de parasitismo total fue de $31.7 \%$ en ambos sitios, lo cual genera un potencial de uso en programas de control biológico por conservación.

Palabras clave: Braconidae, Diapriidae, Bombyliidae, Hemipenthes, nuevos registros

Muñiz-Reyes, E., J. R. Lomelí-Flores \& J. Sánchez-Escudero. 2011. Native parasitoids of Rhagoletis pomonella Walsh (Diptera: Tephritidae) in hawthorn in central Mexico. Acta Zoológica Mexicana (n. s.), 27(2): 425-440.

ABSTRACT. Rhagoletis pomonella is one of the main pests of hawthorn in central Mexico. This pest can damage $100 \%$ fruit when no control tactics are applied. A survey from 2007 to 2009 in Texcoco, Mexico, was held in order to explore the natural enemy complex of this pest. Puparia of Rhagoletis pomonella were collected in soil from infested hawthorn trees. Puparia were kept under controlled conditions during diapause awaiting for parasitoid emergence. 2734 puparia were collected, but only 1460

Recibido: 09/09/2010; aceptado: 01/02/2011. 
insects emerged: $68.3 \%$ of them were fruit flies and $31.7 \%$ were parasitoids. Six parasitoid species were identified: Coptera sp. (Diapriidae), the most common parasitoid ( $25 \%$ of total parasitism), represents a new record of this species attacking $R$. pomonella pupae. Utetes near canaliculatus (Braconidae) (3.4\%) was previously recorded as a parasitoid of this fruit fly in Mexico; Diachasmimorpha mexicana (Braconidae) $(2.3 \%)$ had not been reported for this area.. Hemipenthes blanchardiana (Bombyliidae) $(<0.5 \%)$ represents the first record of this family attacking $R$. pomonella worldwide. Finally, an individual Ichneumonidae and Braconidae were found, but these were not identified. Total parasitism was $31.7 \%$, this result can generate a potential for the use of these parasitoids in conservation biological control programs.

Key words: Braconidae, Diapriidae, Bombyliidae, Hemipenthes, new records

\section{INTRODUCCIÓN}

Rhagoletis pomonella Walsh se encuentra ubicada dentro la familia Tephritidae, comúnmente conocida como moscas de la fruta. Yee \& Goughnour (2008), señalan que las poblaciones geográficamente aisladas de esta plaga han evolucionado para explotar diferentes hospedantes. En México esta especie se encuentra asociada a rosáceas, principalmente al cultivo de tejocote (Crataegus spp.) y manzano (Malus pumila Miller) (Bush 1966, Feder 1995, Hernández 2004, Rull 2009); en Estados Unidos y Canada se reportan infestaciones en durazno (Prunus pérsica L.), pera (Pyrus sp.), ciruela (Prunus domestica L.) e incluso se ha asociado a rosas cultivadas (Bush 1966). El daño que provoca esta plaga es directo al fruto al alimentarse de la pulpa, ocasionando que en lugares donde no hay un manejo, la infestación llegue a afectar hasta el $100 \%$ de los frutos. El tejocote es un cultivo de importancia económica en ciertas regiones del centro de México y en otras zonas de pequeña producción, no se tienen sistemas de manejo de esta plaga, por lo que es común que se presenten pérdidas cercanas al $100 \%$ de los frutos por el ataque de esta plaga (Hernández-Ortíz et al. 2004).

Algunos estudios se han enfocado a determinar los patrones de distribución temporal y espacial de $R$. pomonella (Rull et al. 2006) así como la diversidad y abundancia de sus parasitoides (Rull et al. 2009) lo cual ha ayudado al entendimiento de cómo ocurren las asociaciones entre parasitoides y hospedantes. Sin embargo, no existen trabajos que hayan cuantificado el impacto de enemigos naturales en las poblaciones de $R$. pomonella en las zonas de producción de tejocote. A nivel mundial se han reportado al menos 18 especies de himenópteros asociados a $R$. pomonella repartidos en seis familias, de las cuales Braconidae (nueve especies) y Diapriidae (cinco especies) aportan el mayor número de especies (Cuadro 1).

Los trabajos respecto a los parasitoides nativos presentes en poblaciones mexicanas de $R$. pomonella infestando tejocote son escasos, sólo se reconocen cuatro especies de Braconidae que son parasitoides de larva-pupa (Cuadro1). Rull et al. (2009), exploraron los parasitoides de $R$. pomonella en tejocote Crataegus spp. a través del Eje Volcánico Trans-Mexicano, la Sierra Madre Oriental en México y Estados Uni- 
Cuadro 1. Especies de parasitoides reportados atacando a Rhagoletis pomonella.

\begin{tabular}{|c|c|c|c|}
\hline $\begin{array}{c}\text { Familia/especie de } \\
\text { parasitoide }\end{array}$ & $\begin{array}{l}\text { Biología del } \\
\text { parasitoide }\end{array}$ & País reportado & Referencias \\
\hline \multicolumn{4}{|l|}{ Braconidae } \\
\hline $\begin{array}{l}\text { Aphaeretea muscae } \\
\text { Ashmead }\end{array}$ & No disponible & No disponible & $(12),(7)$ \\
\hline $\begin{array}{l}\text { Diachasma alloeum } \\
\text { (Muesebeck) }\end{array}$ & Larva-pupa/END & E.U. y Canadá & $\begin{array}{l}(12),(14),(3),(10), \\
(8),(4),(1)\end{array}$ \\
\hline $\begin{array}{l}\text { Diachasma ferrugineum } \\
\text { (Gahan) }\end{array}$ & Larva-pupa/END & E. U. & $(3),(10),(8)$ \\
\hline $\begin{array}{l}\text { Diachasmimorpha mellea } \\
\text { (Gahan) }\end{array}$ & Larva-pupa/END & E.U. y México & $\begin{array}{l}(12),(14),(10),(8), \\
(15),(5),(2),(16)\end{array}$ \\
\hline $\begin{array}{l}\text { Diachasmimorpha mexicana } \\
\text { (Cameron) }\end{array}$ & Larva-pupa/END & México & $(15)$ \\
\hline Opius downesi Gahan & Larva-pupa/END & E.U. y México & $(12),(8),(1),(5)$ \\
\hline $\begin{array}{l}\text { Utetes canaliculatus } \\
\text { (Gahan) }\end{array}$ & huevo-larva/END & E.U., Canadá y México & $\begin{array}{l}\text { (14), (3), (8), (1), (15) } \\
(9),(13)\end{array}$ \\
\hline Utetes lectoides (Gahan) & Larva /END & E.U. & $(12),(1)$ \\
\hline Utetes richmondi (Gahan) & Larva-pupa/END & E.U. & $(12)$ \\
\hline \multicolumn{4}{|l|}{ Diapriidae } \\
\hline $\begin{array}{l}\text { Coptera cingulatae } \\
\text { Muesebeck }\end{array}$ & Pupa/ END & E.U. & (17) y (11) \\
\hline $\begin{array}{l}\text { Coptera pomonellae } \\
\text { Muesebeck }\end{array}$ & Pupa/ END & E.U. & (17) y (11) \\
\hline Coptera evansi Muesebeck & Pupa/ END & E.U. & (17) y (11) \\
\hline $\begin{array}{l}\text { Coptera occidentalis } \\
\text { Muesebeck }\end{array}$ & Pupa/ END & E.U. & (17) y (11) \\
\hline Psilus sp. & Pupa/END & E.U. y Canadá & $(3),(8)$ \\
\hline Eulophidae & & & \\
\hline $\begin{array}{l}\text { Tetrastichus sp. } \\
\text { Mymaridae }\end{array}$ & Larva/ECT & E.U. & $(3),(5)$ \\
\hline $\begin{array}{l}\text { Patasson conotracheli } \\
\text { (Girault) }\end{array}$ & Huevos/END & E.U. & (12) \\
\hline Pteromalidae & & & \\
\hline $\begin{array}{l}\text { Pteromalus sp. } \\
\text { Ichneumonidae }\end{array}$ & Larva-ECT & Estados Unidos & (5) \\
\hline $\begin{array}{l}\text { Phygadeuon wiesmanni } \\
\text { Sachtl }\end{array}$ & No disponible & $\begin{array}{l}\text { Europa (cría), liberado } \\
\text { en E.U. }\end{array}$ & (6) \\
\hline
\end{tabular}

${ }^{1}$ AliNiazee (1985), ${ }^{2}$ Baranowski et al. (1993), ${ }^{3}$ Cameron \& Morrison (1977), ${ }^{4}$ Glas \& Vet (1983), ${ }^{5}$ Gut \& Brunner (1994), ${ }^{6}$ Hagley et al. (1993), ${ }^{7}$ Kandybina (1977), ${ }^{8}$ Maier (1981), ${ }^{9}$ Monteith (1971), ${ }^{10}$ Monteith (1977), ${ }^{11}$ Muesbeck (1980), ${ }^{12}$ Narayanan \& Chawla (1962), ${ }^{13}$ Prokopy \& Webster (1978), ${ }^{14}$ Rivard (1967), ${ }^{15}$ Rull, et al.(2009), ${ }^{16}$ Wharton \& Marsh (1978) y ${ }^{17}$ Wharton (2007).

*ECT=Ectoparasitoide; $\mathrm{END}=$ Endoparasitoide. 
dos, en este estudio se determinaron los porcentajes de parasitismo y la diversidad de los ensambles; sin embargo, sólo se obtuvieron parasitoides a partir de larvas colectadas de frutos infestados. $R$. pomonella es un tefrítido que presenta una etapa diapáusica en estado pupal (Dean \& Chapman 1973, Boller \& Prokopy 1976) y en este estado de desarrollo se mantiene expuesta a enemigos naturales por un período más prolongado y tal vez estos pudieran tener un efecto más importante en la dinámica de la plaga, por lo que es importante realizar la exploración en estado de pupa. En adición a lo anterior, es posible integrar la exposición de pupas formadas de larva las cuales pudieron estar expuestas a parasitoides de larva y después las que no hayan sido parasitadas en larva puedan ser encontradas por parasitoides de pupa como Coptera spp. (Menezes et al. 1998).

Considerando que la correcta identificación y conocimiento de las relaciones que guardan las plagas y sus enemigos naturales permite establecer en primera instancia la conservación de los mismos, además de potenciar su uso como un recurso natural local con la finalidad de disminuir las poblaciones de la plaga, y que la búsqueda de enemigos naturales en todas las etapas de desarrollo, permite sugerir estrategias de conservación y manejo en el agroecosistema en el que están presentes. Este trabajo tuvo por objetivo explorar la ocurrencia de parasitoides de $R$. pomonella e identificar las especies presentes, así como la determinar el porcentaje de parasitismo natural en dos zonas de Texcoco, México, durante todas las etapas de desarrollo de estados inmaduros de $R$. pomonella.

\section{MATERIALES Y MÉTODOS}

Sitio de colecta. Los sitios de colecta de parasitoides asociados a $R$. pomonella fueron San Miguel Tlaixpan y el Banco de Germoplasma de Crataegus spp. de la Universidad Autónoma Chapingo. La comunidad de San Miguel Tlaixpan (SMT), está situada en la parte central de la parte montañosa al este de Texcoco $\left(19^{\circ} 31^{\prime}\right.$ Latitud Norte; 9849' Longitud Oeste; 2379 m snm) es considerada como la comunidad representativa del somontano (Pérez 2009). En esta comunidad el tejocote junto con otras especies frutales y ornamentales está establecido como cultivo de traspatio para autoconsumo y venta local. El Banco de Germoplasma de Crataegus spp. (BGC) de la Universidad Autónoma Chapingo está ubicado en Lomas de San Juan dentro del Campo Agrícola de esta Universidad (19 ${ }^{\circ} 29^{\prime}$ Latitud Norte; $98^{\circ} 53^{\prime}$ Longitud Oeste; $2250 \mathrm{~m} \mathrm{snm}$ ) (García 1988); éste fue establecido hace 26 años y tiene una colección de 91 accesiones, de los cuales 50 son provenientes de Chiapas, 18 del Estado de México, y 23 de Puebla (Nieto 2007, Com. Pers. ${ }^{1}$ ).

\footnotetext{
${ }^{1}$ Dr. Raúl Nieto-Ángel. Profesor-Investigador Titular. Universidad Autónoma Chapingo. Departamento de Fitotecnia.
} 
Muestreo preliminar. Durante el mes de enero y febrero de 2007 se realizaron muestreos preliminares de suelo en el Banco de Germoplasma, lo anterior para establecer la metodología de acuerdo a las condiciones del lugar y de la disposición de árboles y frutos de Crataegus. En este período se realizaron tres colectas (enero-febrero de 2007). En cada visita se colectaron al menos 200 puparios. Los puparios colectados se procesaron como se indica más adelante y se llevaron a una cámara de cría $\left(25 \pm 2^{\circ} \mathrm{C} ; 60 \pm 10\right.$ HR; 14:10 L:O) para esperar la emergencia tanto de moscas como de parasitoides.

Muestreos exploratorios. Se realizaron siete muestreos de suelo en el Banco de Germoplasma desde septiembre de 2008 a julio de 2009; mientras que en San Miguel Tlaixpan fueron seis colectas de diciembre de 2008 a julio de 2009. El tamaño de muestra fue variable y dependió de la disponibilidad de pupas en el sitio seleccionado; sin embargo, se tomó como tamaño de muestra el número de puparios colectados en $10 \mathrm{~h}$, debido a que la disposición de árboles, la distribución y variedad de árboles en las zonas de colecta fue muy variable.

Obtención del material biológico. La colecta de puparios se realizó bajo la zona de goteo de los árboles de tejocote. Se utilizó una pala para remover el suelo y la vegetación en la zona de colecta. De acuerdo a lo reportado por Aluja (1993), las pupas se buscaron en los primeros diez centímetros de profundidad. La colecta se realizó mediante el uso de una palita de mano con la cual se removió el suelo por partes (cada $10 \mathrm{~cm}$ aproximadamente) para no lastimar a los puparios que pudieran estar en esa zona, con unas pinzas sin punta aguda se tomaron los puparios que posteriormente se depositaron en viales de vidrio de $25 \mathrm{~mL}$ con suelo del sitio de colecta. Posteriormente se trasladaron al laboratorio de Control Biológico del Colegio de Postgraduados en Montecillo, México, para su procesamiento y esperar la emergencia de los parasitoides y/o moscas. Es importante señalar que se decidió colectar puparios en el suelo, debido a que se asumió que los parasitoides tuvieron oportunidad de llegar a todos los estados inmaduros de $R$. pomonella.

Las pupas colectadas en campo pasaron por un proceso de desinfección externa con una solución de hipoclorito de sodio al 5\%, posterior a ello se colocaron en grupos de 10 confinadas en cajas petri desechables $(5 \mathrm{~mm})$ con almohadillas de algodón humedecido con $2 \mathrm{~mL}$ de agua destilada y sobre ésta se colocó papel filtro (No. 4). Todo el material utilizado fue esterilizado para evitar contaminación por bacterias $\mathrm{u}$ hongos saprófitos. Posteriormente se mantuvieron en la cámara de cría del laboratorio de Entomología del CP, bajo las condiciones arriba señaladas.

Los puparios de $R$. pomonella se revisaron cada tercer día para esperar la emergencia de parasitoides. En caso de contaminación en el papel filtro los puparios fueron transferidos a nuevas cajas de Petri con las condiciones arriba señaladas. El seguimiento de emergencias se registro en una bitácora, anotando los cambios que sucedieron en cada uno de los puparios y cuando se dio el caso de muerte de alguno de ellos, se aisló y determinó la causa. 
En noviembre de 2009 se concluyo el seguimiento ya que las emergencias se redujeron a cero en el último mes. Las pupas de las cuales no se obtuvieron parasitoides ni moscas fueron disecadas para determinar su contenido. Todos los organismos emergidos fueron colectados en alcohol al $70 \% \mathrm{y}$ algunos ejemplares se montaron para su identificación.

Identificación del material biológico. Los adultos emergidos tanto de moscas como de parasitoides se separaron por sexo para registro, montaje y posterior identificación. Los adultos de las moscas fueron identificados usando la descripción de Aluja (1993) y una muestra fue enviada al especialista del grupo (Dr. Vicente Hernández Ortiz, Instituto de Ecología, A. C. Xalapa, Veracruz) para la corroboración de la especie.

Los parasitoides se montaron en triángulo de cartón y algunos ejemplares se prepararon para fotografía en Microscopio Electrónico de Barrido JEOL-6390, mientras que otros se procesaron para fotografía en un Microscopio Tessovar con una cámara digital para microscopía PaxCam 3.

La identificación preliminar de los parasitoides se realizó mediante el estudio de los caracteres morfológicos utilizando las claves de Wharton \& Marsh (1978) y Wharton (2007) para Bracónidos; Muesebeck (1980) y Montilla \& García (2008) para diapríidos y las provistas por Ávalos-Hernández (2007) y Yao \& Yang (2008) para Bombyliidae.

Ejemplares de resguardo de $R$. pomonella y de los parasitoides se depositaron en la Colección de Insectos del Colegio de Postgraduados. Un duplicado de los diferentes grupos de parasitoides fue enviado para la identificación con especialistas de los diferentes grupos. Los bracónidos fueron enviados al Dr. Wharton de la Universidad de Texas A\&M y la Dra. Larissa Guillén de Instituto de Ecología A. C., México; los diapríidos al Dr. Mattew Yoder del Departamento de Entomología de la Universidad de Ohio, Dr. García y Rafael Montilla, del Museo del Instituto de Zoología Agrícola (MIZA) de la Facultad de Agronomía de la Universidad Central de Venezuela (UCV). Finalmente, los bombílidos se enviaron al M. C. Omar Ávalos-Hernández del Museo de Zoología de la Universidad Nacional Autónoma de México.

Determinación del porcentaje de parasitismo. El porcentaje de parasitismo se calculó como lo señala Rull et al. (2009) en el estudio sobre diversidad y abundancia relativa de parasitoides himenópteros de poblaciones mexicanas de $R$. pomonella en tejocote, para lo cual se consideró el número de parasitoides emergidos en relación al total de especímenes emergidos (parasitoides más adultos de $R$. pomonella). Durante la espera de emergencia, en algunas pupas se observó crecimiento de micelios, cuando esto ocurrió se disecaron los puparios y se realizaron preparaciones temporales para observar las estructuras en un microscopio compuesto. De esta manera se determinó si se trataba de un hongo entomopatógeno o saprófito. Aunado a esto, se disecaron puparios en los que finalmente no se obtuvo emergencia de moscas o parasitoides para determinar su contenido. 


\section{RESULTADOS Y DISCUSIÓN}

En las colectas preliminares (enero-febrero de 2007, Banco de Germoplasma) se colectaron 640 puparios de $R$. pomonella de las cuales emergieron 84 moscas y 22 parasitoides pertenecientes a tres especies. Mientras que en el período de exploración (septiembre 2008 a julio 2009, Banco de Germoplasma y San Miguel Tlaixpan) se colectaron 2734 puparios de $R$. pomonella, de los cuales emergieron 997 moscas y 462 parasitoides clasificados en cinco especies distintas (Cuadro 1 y 2).

Cuadro 2. Parasitoides nativos de Rhagoletis pomonella Walsh asociados a tejocote en el Banco de Germoplasma de Crataegus spp., Texcoco, México.

\begin{tabular}{|c|c|c|c|c|}
\hline $\begin{array}{l}\text { Fecha de } \\
\text { colecta }\end{array}$ & $\begin{array}{l}\text { No. total } \\
\text { de pupas } \\
\text { colectadas }\end{array}$ & $\begin{array}{l}\text { Total organismos } \\
\text { emergidos }\end{array}$ & Especies emergidas & $\begin{array}{c}\text { Total }(\% \\
\text { emergencia })^{*}\end{array}$ \\
\hline \multirow[t]{4}{*}{ Sep-08 } & 380 & 196 & R. pomonella & $151(77.0 \%)$ \\
\hline & & & Coptera sp. & $26(13.3 \%)$ \\
\hline & & & D. mexicana & $9(4.6 \%)$ \\
\hline & & & $U$. near canaliculatus & $10(5.1 \%)$ \\
\hline \multirow[t]{3}{*}{ Oct-08 } & 532 & 219 & R. pomonella & $189(86.3 \%)$ \\
\hline & & & Coptera sp. & $21(9.6 \%)$ \\
\hline & & & D. mexicana & $9(4.1 \%)$ \\
\hline \multirow[t]{3}{*}{ Nov-09 } & 401 & 181 & R. pomonella & $154(85.0 \%)$ \\
\hline & & & Coptera sp. & $25(13.8 \%)$ \\
\hline & & & D. mexicana & $2(1.1 \%)$ \\
\hline \multirow[t]{5}{*}{ Dic-08 } & 215 & 129 & R. pomonella & $108(83.1 \%)$ \\
\hline & & & Coptera sp. & $18(13.8 \%)$ \\
\hline & & & D. mexicana & $2(1.5 \%)$ \\
\hline & & & U. near canaliculatus & $1(0.8)$ \\
\hline & & & Hemipenthes & $1(0.8 \%)$ \\
\hline \multirow[t]{2}{*}{ Mar-09 } & 32 & 26 & R. pomonella & $22(84.6 \%)$ \\
\hline & & & Coptera sp. & $4(15.4)$ \\
\hline \multirow[t]{3}{*}{ May-09 } & 39 & 32 & R. pomonella & $25(78.1 \%)$ \\
\hline & & & Coptera sp. & $4(12.5 \%)$ \\
\hline & & & D. mexicana & $3(9.4 \%)$ \\
\hline \multirow[t]{4}{*}{ Jul-09 } & 43 & 40 & R. pomonella & $34(85 \%)$ \\
\hline & & & Coptera sp. & $2(5.0 \%)$ \\
\hline & & & D. mexicana & $2(5.0 \%)$ \\
\hline & & & H. blanchardiana & $2(5.0 \%)$ \\
\hline
\end{tabular}

* E1 \% de parasitismo fue calculado considerando solo los organismos emergidos (R. pomonella + parasitoides). 
Del total de puparios de $R$. pomonella colectados en el muestreo preliminar, el $83.5 \%$ sufrió daño debido al manejo, y sólo la emergieron 22 parasitoides adultos: cuatro ejemplares de Diachasmimorpha mexicana (Cameron) (Braconidae), $10 \mathrm{de}$ Coptera sp. (Diapriidae) y ocho de Hemipenthes blanchardiana Jaennicke (Bombyliidae).

En los muestreos de exploración, de un total de 2734 puparios colectados en ambos sitios sólo emergieron 1460 insectos, de los cuales 68.3\% fueron moscas (997 ejemplares) con un parasitismo total de $31.7 \%$ (463 ejemplares). Coptera sp. fue el parasitoide más abundante con 373 ejemplares (25.5\% del parasitismo total), seguido de Utetes near canaliculatus (Gahan) con 49 ejemplares (3.4\%) y D. mexicana, con 33 ejemplares $(2.3 \%)$ el resto de las especies representaron menos del $0.4 \%$ del total de parasitismo. En ambos sitios se colectó un mayor número de puparios al reportado; sin embargo, muchos de estos no se consideraron en el análisis ya que se hallaron vacios. En los Cuadros 2 y 3 se pueden observar los porcentajes obtenidos para cada una de las fechas de colecta en ambos sitios.

En el muestreo exploratorio en el Banco de Germoplasma se obtuvieron 1642 puparios de los cuales emergieron 825 organismos, 683 fueron adultos de moscas (82.8\%) por lo que el parasitismo total fue de $17.2 \%$ (142 ejemplares) Coptera $\mathrm{sp}$. representó el $12.2 \%$ del parasitismo total con 100 ejemplares, y fue más abundante en las colectas de septiembre a diciembre (2008). La segunda especie en abundancia fue $D$. mexicana, con 27 especímenes (3.3\% de parasitismo). $U$. near canaliculatus representó $1.3 \%$ con 11 ejemplares. Por último, H. blanchardiana y el ichneumónido (no identificado) representaron menos del $0.4 \%$.

En San Miguel Tlaixpan se colectaron un total de 1092 puparios, el total de organismos emergidos fue de 635, de éstos el $49.4 \%$ fueron moscas (314 ejemplares) por lo que el porcentaje total de parasitismo fue de 50.6\% (321 parasitoides). Coptera $\mathrm{sp}$. fue el parasitoide más abundante con 276 especímenes, lo que correspondió al 43.5\% de parasitismo total. $U$. near canaliculatus ocupó el segundo lugar en abundancia y representó el 6\% de los parasitoides emergidos (38 ejemplares). El resto de las especies (Diachasmimorpha mexicana y una especie de Braconidae (No identificado) representaron $1.1 \%$ de parasitismo total.

\section{Diapriidae. Coptera.}

Los miembros de la familia Tephritidae son los hospedantes más comunes de las especies de Coptera; sin embargo, otros dípteros de las familias Psilidae, Muscidae, Milichiidae, Otitidae, Drosophilidae y Lonchaeidae han sido reportados como hospedantes de estos endoparasitoides de pupas (Aguiar-Menezes et al. 2003, Muesebeck 1980). Para el género Rhagoletis existen reportes de Coptera cingulatae (Mues.), C. pomonellae (Mues.), y C. occidentalis (Mues.) como parasitoides de puparios (Mue- 
Cuadro 3. Parasitoides nativos de Rhagoletis pomonella Walsh asociados a tejocote Crataegus spp. en la comunidad de San Miguel Tlaixpan, Texcoco, México.

\begin{tabular}{|c|c|c|c|c|}
\hline $\begin{array}{l}\text { Fecha de } \\
\text { colecta }\end{array}$ & $\begin{array}{l}\text { No. total } \\
\text { de pupas } \\
\text { colectadas }\end{array}$ & $\begin{array}{l}\text { Total organismos } \\
\text { emergidos }\end{array}$ & Especies emergidas & $\begin{array}{c}\text { Total }(\% \\
\text { emergencia }) *\end{array}$ \\
\hline \multirow[t]{3}{*}{ Dic-08 } & 143 & 116 & R. pomonella & $49(42.2 \%)$ \\
\hline & & & Coptera sp. & $65(56 \%)$ \\
\hline & & & $U$. near canaliculatus & $2(1.7 \%)$ \\
\hline \multirow[t]{4}{*}{ Ene-09 } & 151 & 128 & R. pomonella & $50(39.1 \%)$ \\
\hline & & & Coptera sp. & $71(55.4 \%)$ \\
\hline & & & D. mexicana & $2(1.5 \%)$ \\
\hline & & & $U$. near canaliculatus & $5(3.9 \%)$ \\
\hline \multirow[t]{4}{*}{ Feb-09 } & 267 & 118 & R. pomonella & $47(39.8 \%)$ \\
\hline & & & Coptera sp. & $58(49.1 \%)$ \\
\hline & & & D. mexicana & $1(0.8 \%)$ \\
\hline & & & U. near canaliculatus & $12(10.2 \%)$ \\
\hline \multirow[t]{4}{*}{ Mar-09 } & 408 & 167 & R. pomonella & $82(49.1 \%)$ \\
\hline & & & Coptera sp. & $67(40.1 \%)$ \\
\hline & & & D. mexicana & $1(0.5 \%)$ \\
\hline & & & $U$. near canaliculatus & $17(10.2 \%)$ \\
\hline \multirow[t]{4}{*}{ Jun-09 } & 60 & 56 & R. pomonella & $45(78.9 \%)$ \\
\hline & & & Coptera sp. & $10(17.5 \%)$ \\
\hline & & & D. mexicana & $1(1.8 \%)$ \\
\hline & & & Braconidae morph.sp2 & $1(1.8 \%)$ \\
\hline \multirow[t]{2}{*}{ Jul-09 } & 63 & 47 & R. pomonella & $41(87.2 \%)$ \\
\hline & & & Coptera sp. & $5(10.6 \%)$ \\
\hline
\end{tabular}

El \% de parasitismo fue calculado considerando solo los organismos emergidos ( $R$ pomonella + parasitoides).

sebeck 1980); por otra parte Hagen et al. (1981) reportan, a partir de la exposición a los parasitoides $C$. evansi (Mues.) y C. occidentalis (Mues.) en laboratorio a puparios de Rhagoletis completa Cresson como hospedantes. Muesebeck (1980) describe nuevas especies y cuatro de éstas son parasitoides de $R$. pomonella (C. cingulatae, $C$. pomonellae, C. evansi, y C. occidentalis).

Los ejemplares colectados en el presente estudio correspondieron a una sola morfoespecie. De acuerdo con la revisión de las claves taxonómicas de los diferentes autores ya mencionados, las características morfológicas de los ejemplares colectados, tanto de hembras como de machos, no correspondieron con ninguna de las especies reportadas como parasitoides de $R$. pomonella y tampoco con las descritas por Mue- 
sebeck (1980) quien realizó el último trabajo sobre taxonomía del género en la región Neártica. Por otra parte, para las especies del Neotrópico descritas por Montilla \& García (2008) y por Loiácono (1981) tampoco coincidieron las características de los ejemplares obtenidos. Másner \& García (2002) mencionan que es posible que existan 150 especies de este género en el Neotrópico, la mayoría aun sin describir. Es posible que suceda lo mismo con las especies mexicanas ya que se han hecho muy pocas exploraciones de este grupo. Los especímenes se encuentran en proceso de determinación por los especialistas del género, y es muy probable que no sólo se trate de una especie no reportada para $R$. pomonella, (Cuadro 1) sino que también puede tratarse de una especie no descrita por lo que el hallazgo constituye un nuevo reporte para este hospedante y posiblemente una nueva especie para la ciencia.

El porcentaje de parasitismo total atribuible a Coptera en San Miguel Tlaixpan (43.5\%) fue mayor al encontrado en el Banco de Germoplasma (12.2\%). La diferencia pudiera deberse a las condiciones ambientales, tipo y condiciones de suelo y diversidad en especies vegetales. Por ejemplo en las huertas de traspatio de San Miguel Tlaixpan, existe una alta diversidad vegetal, y las plantas reciben un aporte más constante de agua, lo que podría contribuir a generar estabilidad en la población de este parasitoide; mientras que en el banco de germoplasma existen muchas accesiones de Crataegus spp., se trata de un monocultivo, y las aportaciones adicionales de agua son escasa y/o nulas, restando pues las condiciones adecuadas para el establecimiento de mayor cantidad de individuos de este diapriido.

\section{Braconidae}

En América existen reportes de un gran número de especies de bracónidos de la subfamilia Opiinae atacando tefrítidos (Wharton \& Marsh 1978) y se han reportado al menos nueve especies distribuidas en cinco géneros atacando larvas de $R$. pomonella.

\section{Diachasmimorpha}

El género incluye especies de las regiones Neárticas y Neotropicales del Norte, IndoAustraliana y Afrotropical (Wharton 2007). Algunas especies de este género estaban situadas en los géneros Opius y Biosteres y fueron transferidas al género Diachasmimorpha por Van Achterberg \& Mateo en 1990 (Wharton 2007). Este género ha sido reportado atacando tefrítidos sobre todo en el género Anastrepha spp., dentro de éstos se incluye la especie exótica D. longicaudata (Wharton et al. 1981, Aluja et al. 1990, López et al. 1999, Hernández-Ortiz 2006). Las especies reportadas en asociación con R. pomonella son Diachasmimorpha mellea (Gahan) (Wharton \& Marsh 1978) (sin. Biosteres rhagoletis Richmond) (Richmond 1915) y Diachasmimorpha mexicana (Rull et al. 2009). Ambas especies están ubicadas dentro del grupo mexicana, la pri- 
mera se reporta como hospedante $R$. pomonella y está asociada a plantas en el género Crataegus spp. (Maier 1981, Gut \& Brunner 1994, Stelinski et al. 2004). Para el caso de D. mexicana, Wharton (2007) menciona que no existen registros de hospedantes; sin embargo en 2009, Rull et al. obtuvieron cuatro ejemplares de puparios de $R$. pomonella a partir de frutos infestados en Hidalgo y Puebla, y reportan un parasitismo de $1.8 \%$ y $4.3 \%$ respectivamente. En ese mismo trabajo se realizaron colectas en Texcoco, pero no se obtuvo la emergencia de ningún parasitoide.

Diachasmimorpha mexicana ocupó el segundo lugar en abundancia en el Banco de Germoplasma, con 3.3\% de parasitismo (27 ejemplares) mientras que en San Miguel Tlaixpan fue menos abundante con $0.9 \%$ (6 ejemplares). Estos niveles de parasitismo son parecidos a los obtenidos por Rull et al. (2009) en el único trabajo sobre parasitoides de $R$. pomonella en México. El número de parasitoides colectados en los meses de exploración en ambos sitios, fue bajo (en promedio 2.5 individuos/colecta) con excepción de Coptera (en promedio 29 individuos/colecta), que se colectó en todos los meses de muestreo.

\section{Utetes (Gahan) (=Opius)}

Estos miembros de Braconidae son de distribución mundial, y sólo un cuarto de las especies de este género han sido descritas y se han obtenido a partir de especies de tefrítidos. En las regiones Neárticas y Paleárticas, Rhagoletis se presenta como un importante hospedante; sin embargo, también se reportan especies de Utetes atacando a diversas familias de Diptera como Agromyzidae y Anthomyiidae (Wharton 2007).

Fischer (1972) reconoció a éste como subgénero de Opius pero Wharton (1988) lo eleva a un rango genérico, sin embargo la mayoría de las especies no se transfirieron a Utetes si no hasta 1997 (Wharton 2007). U. canaliculatus es una especie que se reporta atacando a $R$. pomonella y a otras especies del género como $R$. cornívora, $R$. fausta, $R$. mendax, $R$. tabellariae y $R$. zephyria en E.U. y Canadá (Wharton \& Marsh 1978, CPC 2010).

Rull et al. (2009) reportó a $U$. canaliculatus como una especie compartida biogeográficamente en México y Estados Unidos, por lo que era de esperarse la presencia de esta especie en la zona de colecta. De acuerdo con las claves morfológicas de Wharton \& Marsh (1978), Wharton (2007), y la corroboración por parte del propio Dr. Wharton, la especie no puede determinarse con certeza, de manera que fue identificada como $U$. near canaliculatus (Wharton, Com. Pers., 2010²). Algunos ejemplares están en revisión por el especialista y es probable que se trate de una variante de esta especie o incluso una especie no descrita.

\footnotetext{
${ }^{2}$ Dr. Robert Wharton. Department of Entomology. Texas A\&M University. College of Agriculture and Life Science.
} 
En las colectas realizadas en el Banco de Germoplasma, se obtuvo un menor porcentaje de parasitismo con respecto a las colectas de San Miguel Tlaixpan. El nivel de parasitismo fue de 1.3\% (11 ejemplares), mientras que en San Miguel Tlaixpan fue de $6 \%$ (38 ejemplares), esto puede deberse al efecto positivo de las condiciones ambientales que prevalecen en San Miguel Tlaixpan como se menciona para el caso de Coptera. Del total de las colectas de U. near canaliculatus en el Banco de Germoplasma, el 91\% fue obtenido en septiembre (2008) y sólo un ejemplar se obtuvo en diciembre (2008); mientras tanto, en San Miguel Tlaixpan se obtuvieron ejemplares de esta especie en casi todos los meses de colecta, excepto en junio y julio. El bajo nivel parasitismo también puede estar dado por el hecho de que para el caso de los sitios de colecta mencionados, no se aprecia una competencia entre moscas de Rhagoletis y otras plagas como palomillas o picudos, de manera que las larvas que infestan los tejocotes quedan en la parte más interna de la fruta, logrando reducir el alcance de la oviposición de ambos parasitoides (D. mexicana y $U$. near canaliculatus), aunado a ello el tamaño del ovipositor de estos parasitoides puede no ser suficiente para alcanzar las larvas que se encuentran dentro del fruto, lo cual es referido por Feder (1995), quien describe para este caso en particular un "escape de enemigos naturales".

\section{Bombyliidae (Diptera)}

Los bombílidos pertenecen a la superfamilia Bombylioidea y están relacionados con las familias Asilidae y los Therevidae (Evenhuis 2008). Es una de las familias más diversas dentro del orden Diptera, sin embargo, no existe gran abundancia de individuos (Ávalos 2007) y quizá esta sea la razón de que esta familia sea muy poco estudiada.

\section{Hemipenthes}

El único registro encontrado en la literatura de parasitismo por bombílidos en el género Rhagoletis es Thyridanthrax after sobre R. batava (Kandybina 1977). Por lo que el presente trabajo proporciona el primer registro de Hemipenthes blanchardiana como parasitoide de R. pomonella. En la subfamilia Anthracinae se incluye el género $\mathrm{He}$ mipenthes que tiene una distribución mundial e incluye a 77 especies conocidas (Yao \& Yang 2008). Ávalos-Hernández (2007) realizó colectas de este y otros géneros de bombílidos en una reserva en Morelos y describe a Hemipenthes como organismos que presentan una conducta territorial sobre cuerpos de agua; sin embargo, no existía reporte alguno acerca de la biología de este parasitoide sobre tefrítidos hospedantes.

El hallazgo de la especie $H$. blanchardiana sobre puparios de $R$. pomonella constituye el primer reporte mundial de este parasitoide. En el Banco de Germoplasma en el muestreo preliminar, se obtuvieron ocho ejemplares adultos y la especie fue deter- 
minada por Omar Ávalos-Hernández (2009). Al momento de disecar los puparios se encontraron tres pupas de este bombílido, una en el mes de diciembre y dos en julio, no hubo emergencia de adultos como ocurrió en el muestreo preliminar, esto puede deberse a dos razones, primero, porque este insecto se presenta aen bajas densidades ya sea como parasitoide o hiperparasitoide (Ávalos 2010, Com. Pers. ${ }^{3}$ ) y segundo, los árboles de tejocote en el Banco a partir de la cosecha de octubre 2007 (octubrenoviembre), sufrieron un daño considerable debido a la falta de agua y nutrientes . La falta de humedad pudo afectar de manera importante la viabilidad de los puparios y probablemente $H$. blanchardiana sea una especie sensible a los cambios drásticos, estos efectos de variación ambiental pueden afectar la dinámica poblacional de parasitoides (Rull et al. 2009)

En términos generales, es probable que el porcentaje de parasitismo obtenido en campo esté sobre o subestimado; Hagley et al. (1993), reportó que en los estados de larvas y pupas de Rhagoletis pomonella la mortalidad natural es alta y Monteith (1971 \& 1977), señala que el parasitismo no es un factor de importancia en la mortalidad de larvas y pupas de $R$. pomonella, en ese mismo sentido, Rull et al. (2009) consideran que la mortalidad puede ser causada por un agotamiento en las reservas de energía durante la diapausa antes de la emergencia de moscas, y aunada a esta situación, los parasitoides pueden fracasar para completar su desarrollo y emergencia por diferentes razones. Las condiciones ambientales pueden ejercer también un efecto diferente en cada uno de los organismos, afectando el desarrollo de los parasitoides en mayor medida que el de las moscas, lo cual restringe la emergencia de los parasitoides (Feder 1995). El bajo porcentaje de emergencia de los bracónidos y las nulas emergencias de $H$. blanchardiana en el segundo periodo de colecta pudo deberse precisamente a este tipo de influencias. Otro factor influyente es la variabilidad en los distintos genotipos de árboles de tejocote (Crataegus sp.) en el Banco de Germoplasma y en mucho menor medida también en San Miguel Tlaixpan, que incluye variedades tempranas y tardías y esto, según lo que explica Rull et al. (2009), puede provocar que a los parasitoides se les dificulte coincidir en el tiempo con los estados inmaduros de las moscas en la fruta.

AGRADECIMIENTOS. Al CONACyT-México, por el apoyo para la realización de estudios de posgrado de la primer autor. Al M.C. Omar Avalos de la UNAM por la identificación de la especie $\mathrm{He}$ mipenthes blanchardiana reportada en este trabajo. Al Dr. Robert A. Wharton de la Universidad de Texas A\&M por su apoyo en la identificación de los ejemplares bracónidos. Al Dr. Matt Yoder de la Universidad de Ohio y al Dr. Rafael Montilla de la Universidad de Venezuela por su gran apoyo para lograr la identificación de la especie del género Coptera, la cual sigue en revisión. Al M.C. Jorge Valdés

\footnotetext{
${ }^{3}$ M.C. Omar Ávalos-Hernández. Museo de Zoología. Facultad de Ciencias. Universidad Nacional Autónoma de México.
} 
del Colegio de Postgraduados por su valioso apoyo en la obtención de imágenes que sirvieron para la identificación de las especies de bracónidos, así como a la bióloga Greta Hanako del Laboratorio de Microscopía Electrónica del CP para la obtención de imágenes de barrido en los ejemplares de Coptera.

\section{LITERATURA CITADA}

Aguiar-Menezes, E. L., E. B. Menezes \& M. S. Loiácono. 2003. First record of Coptera haywardi Loiácono (Hymenoptera: Diapriidae) as a parasitoid of fruit-infesting Tephritidae (Diptera) in Brazil. Neotropical Entomology, 32: 355-358.

AliNiazee, M. T. 1985. Opiinae parasitoids (Hymenoptera: Braconidae) of Rhagoletis pomonella and R. zephyria (Diptera: Tephritidae) in the Willamette Valley, Oregon. Canadian Entomologist., 117: 163-166.

Aluja, M. 1993. Manejo Integrado de Moscas de la Fruta. Ed. Trillas. México. 241 p.

Aluja, M., J. Guillen, P. Liedo, M. Cabrera, E. Ríos, G. De la Rosa, H. Celedonio, \& D. Mota. 1990. Fruit infesting tephritids (Diptera: Tephritidae) and associated parasitoids in Chiapas, Mexico. Entomophaga, 35: 39-48.

Ávalos-Hernández, O. 2007. Bombyliidae (Insect: Diptera) de Quilamula en el área de Reserva Sierra de Huautla, Morelos, México. Acta Zoológica Mexicana (n. s.), 23: 139-169.

Baranowski, R. M., H. Glenn \& J. Sivinski. 1993. Biological Control of the Caribbean Fruit Fly (Diptera: Tephritidae). Florida Entomologist, 76: 245-251.

Boller, E. F. \& R. J. Prokopy. 1976. Bionomics and management of Rhagoletis. Annual Review of Entomology, 21: 223-246.

Cameron, P. J. \& F. O. Morrison. 1977. Analysis or mortality in the apple maggot, Rhagoletis pomonella (Diptera: Tephritidae) in Quebec. Canadian Entomologist, 109: 769-787.

Dean, R. W. \& Chapman, P. J. 1973. Bionomics of the apple maggot in Eastern New York, Search Agriculture Entomology. Geneva, N.Y. 3: 62 pp.

Feder, J. L. 1995. The effects of parasitoids on sympatric host races of Rhagoletis pomonella. Ecology, 76: 801-813.

Evenhuis, N. L. 2008. A compendium of zoological type nomenclature: a reference source. USA, Bishop Museum Press, Bishop Museum Technical report 41, 23 pp.

Fischer, M. 1972. Hymenoptera, Braconidae (Opiinae I). Das Tierreich, 91: 1-620.

García, E. 1988. Modificaciones al Sistema de Clasificación Climática de Köppen. Edit. UNAM. D. F., México. 246 pp.

García, J. L. \& R. Montilla. 2001. Coptera haywardi Loiácono (Hymenoptera: Diapriidae) a parasitoid of pupae of Anastrepha spp. (Diptera: Tephritidae) in Venezuela. Entomotropica, 16: 191-195.

Glas, P. C. G. \& L. E. M. Vet. 1983. Host-habitat location and host location by Diachasma alloeum Muesebeck (Hymenoptera: Braconidae), a parasitoid of Rhagoletis pomonella Walsh (Diptera: Tephritidae). Netherlands Journal of Zoology, 33: 41-54.

Gut, L. J. \& J. F. Brunner. 1994. Parasitism of the apple maggot, Rhagoletis pomonella, infesting hawthorns in Washington. Biocontrol, 39: 41-49.

Hagen, K. S., R. L. Tassan \& M. Fong. 1981. Biological control of walnut husk fly. Walnut Research Reports. University of California. Publicación en línea http://walnutresearch.ucdavis. edu/1983/1983_40.pdf (Consultada el 10 de Julio de 2010).

Hagley, E. A. C., A. R. Biggs, G. E. Timbers \& J. Coutu-Sundy. 1993. Effect of age of the puparium of the apple maggot, Rhagoletis pomonella (Walsh) (Diptera: Tephritidae), on parasitism by Phygadeuon wiesmanni Sachtl (Hymenoptera: Ichneumonidae). Canadian Entomologist, 125: 721-724. 
Hernández-Ortiz. V., I. Morales, C. Vergara. 2004. Detección de poblaciones de Rhagoletis pomonella (Diptera: Tephritidae) durante la fructificación de Crataegus mexicana (Rosaceae) en Puebla, México. Acta Zoológica Mexicana (n. s.), 20: 119-129.

Kandybina, M. N. 1977. Lichinki plodovykh mukh-pestrokrylok (Diptera, Tephritidae) (Translated in 1987 by G. Saad. [Larvae of fruit-infesting fruit flies (Diptera, Tephritidae)]. Opredeliteli Po Faune SSSR. No. 114: 1-210.

Loiacono, M. S. 1981. Notas sobre Diapriinae neotropicales (Hymenoptera, Diapriidae). Revista de la Sociedad Entomológica Argentina, 40: 237-241.

López, M., M. Aluja, and J. Sivinski. 1999. Hymenopterous larval-pupal and pupal parasitoids of Anastrepha flies (Diptera: Tephritidae) in Mexico. Biological Control, 15: 119-129.

Maier, C. T. 1981. Parasitoids emerging from puparia of Rhagoletis pomonella (Diptera: Tephritidae) infesting hawthorn and apple in Connecticut. Canadian Entomologist, 113: 867-70.

Másner L. \& J. L. García. 2002. The Genera of Diapriinae (Hymenoptera: Diapriidae) in the New World. Bulletin of the American Museum of Natural History. New York, USA. N² 268, 138 pp.

Menezes, E., J. Sivinski, T. Holler, M. Aluja, F. Jerónimo \& E. Ramírez. 1998. Development of Coptera haywardi (Hymenoptera: Diapriidae) in irradiated and unirradiated pupae of the caribbean fruit fly and the mediterranean fruit fly (Diptera: Tephritidae). Florida Entomologist, 81: 567570.

Monteith, L. G. 1971. The status of parasitoids of the apple maggot Rhagoletis pomonella (Diptera: Tephritidae) in Ontario. Canadian Entomologist, 103: 507-512.

Monteith, L. G. 1977. Additional records and the role of parasitoids of the apple maggot Rhagoletis pomonella (Diptera: Tephritidae) in Ontario. Proceedings of the Entomological Society of Ontario, 108: 3-6.

Montilla, R. \& J. L. García. 2008. Nuevas especies de Coptera Say (Hymenoptera: Proctotrupoidea: Diapriidae: Psilini) para Venezuela. Entomotropica, 23: 43-95.

Muesebeck C. F. W. 1980. The neartic parasitic wasp of the genera Psilus Panzer and Coptera Say (Hymenoptera: Proctotrupoidea: Diapriidae). United States Department of Agriculture, Technical Bulletin, 1617: $71 \mathrm{pp.}$

Narayanan, E. S. \& S. S. Chawla. 1962. Parasites of fruit fly pests of the world with brief notes on their bionomics, habits and distribution. Beiträge zur Entomologie, 12: 437-476.

Pérez, L. M. 2009 (Electrónico). Población y Sociedad: Cuatro comunidades del Acolhuacan. Edit. Universidad Iberoamercana. Edición impresa 1975 INAH. México.

Prokopy, R. J. \& R. P. Webster. 1978. Oviposition deterring pheromone in Rhagolethis pomonella: A kairomone for its parasitoid Opius lectus. Journal of Chemical Ecology, 4: 481-494.

Richmond, E. A. 1915. Biosteres rhagoletis Rhichmond, sp. n., a parasite of Rhagoletis pomonella Walsh. Canadian Entomologist, 47: 293-295.

Rivard, L. 1967. Opius lectus and O. alloeus (Hymenoptera: Braconidae), larval parasites of the apple maggot, Rhagoletis pomonella (Diptera: Tephritidae) in Quebec. Canadian Entomologist, 99: 895896.

Rull, J., M. Aluja, J. L. Feder. \& S. H. Berlocher. 2006. The distribution and host range of hawthorninfesting Rhagoletis (Diptera: Tephritidae) in Mexico. Annals of the Entomological Society of America, 99: 662-672.

Rull, J., R. Wharton, J. L. Feder, L. Guillén, J. Sivinski, A. Forbes \& M. Aluja. 2009. Latitudinal Variation in parasitoid guild composition and parasitism rates of North American hawthorn infesting Rhagoletis. Environmental Entomology, 38: 588-599.

Stelinski, L. L., L. J. Gut, A. V. Pierzchala, \& J. R. Miller. 2004. Field observations quantifying attraction of four tortricid moths to high-dosage pheromone dispensers in untreated and pheromonetreated orchards. Entomologia experimentalis et applicata, 113: 187-196. 
Wharton, R. A. 2007. Parasitoids of fruit-infesting Tephritidae. Publicación en línea http://hymenoptera.tamu.edu/paroffit/ (Consultada el 10 de Julio de 2010).

Wharton, R. A. \& P. M. Marsh. 1978. New world Opiinae (Hymenoptera: Braconidae) parasitic on Tephritidae (Diptera). Journal of Washington Academy of Science, 68: 147-167.

Wharton, R. A., F. E. Gilstrap, R. H. Rhode, M. Fischel \& W. G. Hart. 1981. Hymenopterous eggpupal and larval-pupal parasitoids of Ceratitis capitata and Anastrepha spp. [Dip.: Tephritidae] in Costa Rica. Entomophaga, 26: 285-290.

Yao, G. \& D. Yang. 2008. Two new species of Hemipenthes Loew from Oriental China (Diptera: Bombyliidae). Zootaxa, 1689: 63-68.

Yee, L. W. \& R. B. Goughnour. 2008. Host plant use by and new host records of apple maggot, western cherry fruit fly, and other Rhagoletis species (Diptera: Tephritidae) in western Washington state. Pan-Pacific Entomologist, 84: 179-193. 